\title{
Wireless home automation networks for indoor surveillance: technologies and experiments
}

\author{
Milo Spadacini ${ }^{1,2}$, Stefano Savazzi ${ }^{3^{*}}$ and Monica Nicoli ${ }^{1}$
}

\begin{abstract}
The use of wireless technologies for critical surveillance and home automation introduces a number of opportunities as well as technological challenges. New emerging technologies give the opportunity to exploit the full potential of the internet of things paradigm by augmenting existing wired installations with smart wireless architectures. This work gives an overview of requirements, characteristics, and challenges of wireless home automation networks with special focus on intrusion detection systems. The proposed wireless network is based on several sensors that are deployed over a monitored area for detecting possible risky situations and triggering appropriate actions in response. The network needs to support critical traffic patterns with different characteristics and quality constraints. Namely, it should provide a periodic low-power monitoring service and, in case of intrusion detection, a real-time alarm propagation mechanism over inherently unreliable wireless links subject to fluctuations of the signal power. Following the guidelines introduced by recent standardization, this paper proposes the design of a wireless network prototype at $868 \mathrm{MHz}$ which is able to satisfy the specifications of typical intrusion detection applications. A proprietary medium access control is developed based on the low-power SimpliciTl radio stack (Texas Instruments Incorporated, San Diego, CA, USA). Network performance is assessed by experimental measurements using a test-bed in an indoor office environment with severe multipath and nonline-of-sight propagation conditions. The measurement campaigns highlight the potential of the sub-GHz technology for cable replacing.
\end{abstract}

Keywords: Wireless home automation networks (WHAN); Internet of things; Smart spaces; Wireless sensor networks; Smart surveillance

\section{Introduction}

Wireless sensor network (WSN) technologies are expected to be integrated into the so called internet of things, allowing for the global interconnection of heterogeneous smart objects with advanced functionality. Recent advances of microcontroller design and radio technologies have opened the way to an emerging category of wireless network-enabled sensors that serve as smart agents equipped with a low-power dedicated high-performance microcontroller and a large memory space. Those devices are now gaining the attention of companies operating in the field of smart spaces and advanced surveillance systems. Although most solutions currently on the market are still based on power line or wired communications,

*Correspondence: stefano.savazzi@ieiit.cnr.it

3 Institute of Electronics, Computer and Telecommunication Engineering (IEIIT), Italian National Research Council (CNR), Milan site, c/o DEIB Politecnico di Milano, via Ponzio 34/5, 20133 Milano, Italy

Full list of author information is available at the end of the article it is expected that cable replacing will eliminate timeconsuming installations [1], opening the way to more flexible services for the end user. A wireless home automation network (WHAN) is thus the key enabling technology to make indoor environments intelligent and context aware.

A WHAN is composed by a number of sensors and actuators (e.g., infrared, motion sensors, light switches, safety sensors, accelerometers) that intelligently share their transmission resources and interconnect with each other according to a suitable wireless architecture. As depicted in Figure 1, a network coordinator or access point (AP) is responsible for starting the network, assigning radio resources, monitoring the links' quality, and joining new end devices (ED). ED nodes can be spread over the area of interest (typically indoor and over different floors) while the communication with the AP coordinator might be guaranteed by range extenders (RE) serving as decode and forward relays.

\section{望 Springer}

(c) 2014 Spadacini et al: licensee Springer. This is an Open Access article distributed under the terms of the Creative Commons Attribution License (http://creativecommons.org/licenses/by/2.0), which permits unrestricted use, distribution, and reproduction in any medium, provided the original work is properly cited. 


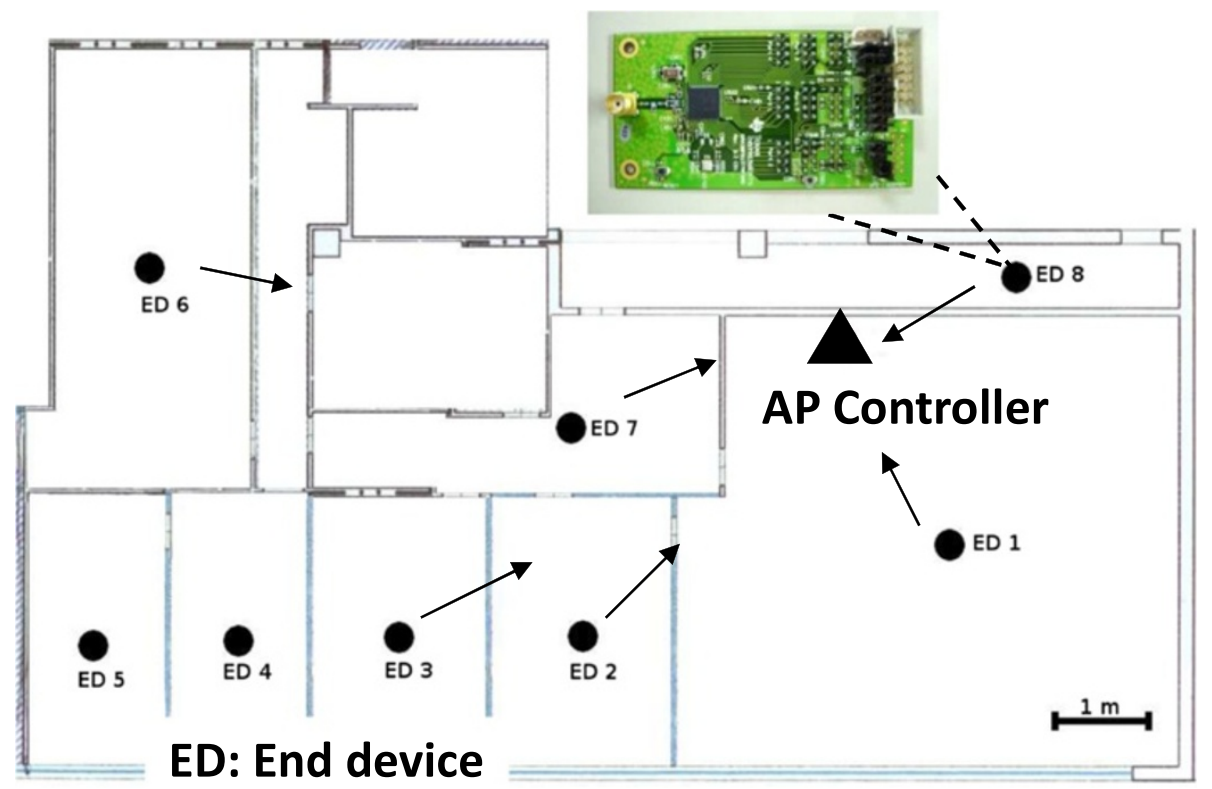

Figure 1 Map of the indoor scenario of application and WHAN deployment with an AP controller and 8 EDs.

A key element for WHAN design and implementation is to provide efficient hardware-software supporting infrastructures and middleware platforms to enable consistent and cost-effective interactions among the diverse actors participating to the smart space environment. Motivated by this vision, in this paper, we consider the design of the medium access control (MAC) for a wireless network supporting critical home automation services, with special focus on indoor surveillance and intrusion detection. The system needs to periodically record activities in the environment and provide a highly reliable connection service for alarm message propagation [2]. The general requirements of wireless intrusion detection systems, evaluated at European level through the standard EN 5013-5-3 [3], are discussed in the first part of the paper. Next, we provide an overview of wireless solutions suited for the selected application, and we propose a performance comparison between two candidate radio technologies operating at $2.4 \mathrm{GHz}$ and $868 \mathrm{MHz}$ based on experimental tests in indoor environments with severe multipath and nonline-of-sight (NLOS) propagation. The measurement campaigns highlight the potential of the sub- $\mathrm{GHz}$ radio technology for home automation as compared to the more conventional $2.4 \mathrm{GHz}$ option. Based on the results of this experimental analysis, we then focus on the sub$\mathrm{GHz}$ radio technology and consider the implementation of the proposed MAC sub-layer on radio modules operating over the $868-\mathrm{MHz}$ ISM band. The guidelines and general rules for the development, and the configuration and the network planning of the WHAN are proposed and tailored to the specific application. The analysis of battery consumption, wireless connectivity, and robustness shows that the proposed system is a promising solution for surveillance and intrusion detection applications.

\section{Requirements and protocols for wireless indoor surveillance}

A WHAN for smart surveillance and intrusion detection has many peculiarities that are common to a broader class of industrial automatic control systems. The physical (PHY) layer radio characteristics mostly common to all these systems are low data rate (below $500 \mathrm{kbps}$ ), carrier frequency at $2.4 \mathrm{GHz}$ or in the $868 / 915-\mathrm{MHz}$ ISM bands, and receiver sensitivity above $-110 \mathrm{dBm}$ [4]. Most of the PHY layer access schemes are based on offset quadrature phase-shift keying (O-QPSK) or frequencyshift keying (FSK) combined with direct-sequence spread spectrum (DSSS) transmission which allows the network susceptibility to interference to reduce, providing a few $\mathrm{dBs}$ of link gain and some improvements over harsh environments characterized by multipath fading. Multi-channel radios are also adopted to efficiently manage the co-channel interference and to reject any external disturbance through dynamic scheduling and channel/frequency hopping.

The basic requirements that need to be considered for WHAN protocol design are listed in the following.

- Network services. A wireless network for smart surveillance must support different classes of traffic patterns with related quality constraints. The network should provide both a periodic low-power monitoring 
service and a real-time alarm propagation mechanism which must be robust enough to cope with inherently unreliable wireless links characterized by signal power fluctuations. Intrusion detection systems have stricter reliability and delay requirements compared to conventional home automation services. Reliable communication occurs only if both sensor observations and feedback from the AP controller are decoded by the respective parties within specified deadlines defined by the controller policy. This hard constraint calls for an advanced wireless link-layer protocol management to provide an optimal trade-off between reliability and real-time communication.

- Indoor radio planning. Indoor home environments are typically characterized by severe multipath due to the presence of reflective surfaces (e.g., walls, floor, and furniture) [4]. Radio planning is a useful tool which relies on the prediction of the wireless link quality. Prediction can be supported by independent radio measurement campaigns over typical indoor buildings and/or by empirical propagation models. A low accuracy in the radio planning design phase will turn into high logistic costs: adding new wireless REs to improve the coverage as well as moving them around the environment may become unacceptable and highly time consuming in some cases.

- Low duty cycling operation. Wireless autonomous devices are battery powered. EDs are usually deployed in predefined spots and must remain active for 3 to 5 years. This poses stringent constraints on the sensor and the radio transceiver design for minimizing the energy consumption. The MAC sub-layer protocol and the application software need to be jointly optimized to preserve the battery. Energy harvesting techniques also provide a powerful tool for lifetime maximization. Some of the techniques employed to reduce power consumption include: (1) dynamic sleep mode activation (with fast wake-up times) to shut-down devices when not transmitting or receiving and (2) low duty cycling design to minimize ED activity cycles. Being the network almost static, the adoption of guaranteed (interference-free) timedivision multiple access (TDMA) and beacon-enabled network designs [5] are to be preferred compared to random access strategies to minimize idle listening.

\subsection{Indoor surveillance systems: EU specifications}

European wireless intrusion-detection systems are governed by the EN 5013-5-3 [3] law that disciplines several aspects like the immunity against channel variations and transmission collisions, the detection of device substitutions, and the robustness to radio interference. The same rules explain how to test every single device to ensure the compliance of the intrusion-detection system to the requirements. The EN 5013-5-3 specifications identify four levels (or grades) of security and specify for each level the requirements on some key system parameters. Below, we briefly describe these parameters and the related requirements for each level of security, ranging from 1 to 4 with increasing security degree.

- Channel immunity. This property is related to the sensitivity of the system to channel variations and particularly to any attenuation increase (i.e., due to deep fading, interference etc.): grade 1 service can support an attenuation increase of up to $3 \mathrm{~dB}$, grade 2 to $6 \mathrm{~dB}$, grade 3 to $9 \mathrm{~dB}$, and grade 4 to $12 \mathrm{~dB}$.

- Transmission collisions. The objective of the collision rate requirement is to ensure a high level of confidence during the transmissions of alarm and monitoring messages to avoid auto-interference (interference among devices of the same system). The collision rate depends on the channel occupation time (or duty cycle) of the devices. Grade 1 service is characterized by a duty cycle larger than $10 \%$ measured over $240 \mathrm{~min}$, duty cycle for grade 2 is lower than $5 \%$ over a period of $240 \mathrm{~min}$ (or $10 \%$ over a period of $120 \mathrm{~min}$ ), while duty cycles for grades 3 and 4 are lower than, respectively, $10 \%$ and $1 \%$ measured over a cycle time period of $100 \mathrm{~s}$. Notice that a more stringent $1 \%$ duty cycle limitation is applied to low-power wireless communications over ISM frequency bands.

- Link reliability. The link reliability measures the probability of information loss during the communication. It is set to $10^{-3}$ for grades 1 to 2 (corresponding to 999 correctly interpreted messages out of 1,000 [3]) and to $10^{-4}$ for grades 3 to 4 (corresponding to 9, 999 correct messages out of 10, 000). For wireless communication over harsh indoor propagation environments, the use of direct/indirect retransmissions (if allowed) can be optimally designed to comply with such specifics.

- Security. In order to prevent both unintentional and intentional device substitution, each transmitter shall be identified by an identification code. The security levels are characterized by the probability for an intruder to discover the identification code in less than $1 \mathrm{~h}$ : this is $5 \%$ for grade $1,1 \%$ for grade $2,0.5 \%$ for grade 3 , and $0.05 \%$ for grade 4 .

- Cross-tier interference. The system robustness against cross-tier radio interference is measured in terms of in-band and out-of-band interference. Let $F_{\min }$ and $F_{\max }$ be the minimum and maximum carrier frequencies: the out-of-band carriers $F_{1}$ and $F_{2}$ are defined as $F_{1}=0.95 \cdot F_{\min }$ and $F_{2}=1.05$. $F_{\text {max }}$. Grades 1 and 2 compliant systems are robust against an interferer centered on $F_{1}$ and $F_{2}$ with an 
intensity of $10 \mathrm{~V} / \mathrm{m}$, while grades 3 and 4 systems are also robust to an in-band interferer centered on the carrier frequency $F_{t}=\left(F_{1}+F_{2}\right) / 2$ with an intensity of $10 \mathrm{~V} / \mathrm{m}$.

- System monitoring. The measurement of the noise and the interference level is implemented by a periodic message exchange. The period interval for link quality sensing depends only on the transmitter role in the system (e.g., ED or RE devices) and on the network topology. From grade 1 to grade 4, the system has to guarantee that the time interval is not greater than $60 \mathrm{~min}, 20 \mathrm{~min}, 100 \mathrm{~s}$, and $10 \mathrm{~s}$, respectively.

- Antenna protection. Grades 1 and 2 are assigned if the antennas cannot be removed without opening the housing, while grades 3 and 4 are assigned if the antennas fulfill the same tamper protection requirements valid for the corresponding devices.

\subsection{Wireless protocols for home and building automation} In the following, a review of the most suitable commercial systems for wireless networking in home and building automation is presented. The selection criteria include frequency bands, data rates, modulation techniques, routing schemes, topologies, interoperability, openness of the software architecture, standardization, and general suitability to support critical home automation applications and security [6].

Bluetooh (Bluetooth Special Interest Group, Kirkland, Washington, USA) and ZigBee (ZigBee Alliance, San Ramon, CA, USA) have been recently investigated in the literature for WHAN applications. A Bluetooth WHAN has been introduced in [7] using a primary network controller and a number of sub-controllers connected by star topology. However, the wireless architecture does not completely replace cabling, and the use of the Bluetooth technology shows disadvantages in terms of access delay. A ZigBee-based WHAN has been proposed in [8]. Although ZigBee interface based on the IEEE 802.15.42006 standard [9] provides an effective network solution for low-power wireless sensing, the overall size of the radio stack (between 45 and $100 \mathrm{~kb}$ ) limits its applications to a small subset of smart home automation scenarios. ZigBee is briefly reviewed in the following together with a selection of wireless technologies that present interesting characteristics for WHAN applications. The comparative analysis is also summarized in Figure 2.

ZigBee is a wireless networking technology developed by ZigBee Alliance for low data rate and short-range applications [8]. Protocol stack is composed of four main layers. The first two layers (PHY and MAC) are defined by the IEEE 802.15.4 standard, while the network (NWK) and application (APL) layers are defined by the ZigBee specifications. The standard IEEE 802.15.4 [9] is a specification for low-power WSN originally designed for the frequency bands 868 to $868.6,902$ to 928 , and 2,400 to 2,485 MHz. For the 2,400 - 2,485 MHz band, the PHY layer transmission maps any 4-bit codeword into a 32-chip sequence using DSSS with a bandwidth expansion factor of 8 . The chip sequences are concatenated, modulated, and translated to radio frequency (RF) using O-QPSK modulation. Today, commercial battery-operated systems enable data to be transmitted at a rate of up to $250 \mathrm{kbps}$, while a maximum power of $12 \mathrm{dBm}$ guarantees a reasonably high channel immunity against deep fades (up to grade 4). In critical environments, the transmit power could not exceed 12 $\mathrm{dBm}$ to meet the RF regulations for the use of unlicensed spectrum in hazardous environments. ZigBee upper layers support two methods for channel access, the beaconless and the beacon-enabled access. In beacon-less mode, devices employ a plain carrier sense multiple access with collision avoidance (CSMA/CA) scheme based on the low power listening principle. The use of CSMA as access technology for all sensors is unsuitable for critical delaysensitive applications such as intrusion detection systems subject to real-time constraints. On the other hand, in beacon-enabled mode, a coordinator node (i.e., the personal area network coordinator) acts as a clock distributor to provide a framing structure by periodically transmitting beacon frames. The frame is the time between two beacons, and it is divided into three parts: a contentionaccess period for CSMA/CA, a contention-free period for TDMA, and an inactive period to power-off devices.

The Z-Wave protocol (Zen Systems, Hillsborough, NJ, USA) [10] was developed with an explicit focus on home control applications. Z-Wave operates at $908 \mathrm{MHz}$ in the US and in the ISM band of $868 \mathrm{MHz}$ in Europe, using FSK modulation with data rate $200 \mathrm{kbps}$. Z-Wave uses a mesh networking approach with source routing, which means that the whole route is determined already at the creation of the frame in the sender. Therefore, only devices which are aware of the entire network topology can send ad hoc messages to any destination. Z-Wave consists of several types of nodes that can be clustered into two main classes, controllers (nodes that create and send control messages) and slaves (nodes that receive and execute the commands). The standard is specifically tailored for remote control of devices used in both residential and commercial buildings. However, the protocol has not been designed to transfer large amounts of data, and it is not suitable for real-time critical data transmission.

EnOcean is a proprietary environment not yet standardized at international level [11]. EnOcean offers its technology and its licenses through the EnOcean Alliance (San Ramon, CA, USA). The objective is to provide selfpowered wireless devices, such as piezoelectric or mini solar panels, highly optimized for energy saving for the automation of homes and buildings. Messages are only a couple of bytes long (with a maximum payload of 6 


\begin{tabular}{|c|c|c|c|c|c|c|}
\cline { 2 - 7 } \multicolumn{1}{c|}{} & $\begin{array}{c}\text { Frequency } \\
\text { band [MHz] }\end{array}$ & $\begin{array}{c}\text { Max. Data-rate } \\
{[\mathrm{Kbps}]}\end{array}$ & Modulation & Security & OpenSource & $\begin{array}{c}\text { Max. Stack } \\
\text { size [KB] }\end{array}$ \\
\hline Zigbee & 2400915868 & 250 & $\begin{array}{c}\text { O-QPSK } \\
\text { BPSK }\end{array}$ & AES -128 & no & 128 \\
\hline Z-Wave & 2400915868 & 200 & $\begin{array}{c}\text { FSK } \\
\text { GFSK }\end{array}$ & $\begin{array}{c}\text { AES-128 (serie } \\
400 \text { only) }\end{array}$ & no & 64 \\
\hline EnOcean & 868 & 125 & ASK & no & no & 24 \\
\hline Wavenis & 915868433 & 100 & $\begin{array}{c}\text { GFSK } \\
\text { PSK }\end{array}$ & $\begin{array}{c}\text { DES-3 } \\
\text { AES -128 }\end{array}$ & no & - \\
\hline MIWI & $\begin{array}{c}2400950915 \\
868\end{array}$ & 200 & FSK & partially yes & 25 \\
\hline Insteon & 904 & 38.4 & FSK & Rolling code & no & 7 \\
\hline KNX-RF & 868.3 & 16.4 & FSK & no & no & - \\
\hline
\end{tabular}

Figure 2 Commercial wireless systems, standards, and software architectures for home and building automation. Green (and yellow) cells indicate desired (and acceptable) features for home automation applications. Red cells highlight critical issues that need to be accounted for during system design.

bytes) and are transmitted using amplitude shift keying (ASK) modulation at the data rate of $125 \mathrm{kbps}$. Packet transmission takes less than $1 \mathrm{~ms}$. The EnOcean protocol cannot increase the transmission reliability by means of end-to-end acknowledgments since its battery-less transmitter modules do not contain a RF receiver. No security mechanisms appear to be included.

Wavenis is a wireless protocol operating at 868,915 , and $433 \mathrm{MHz}$, developed by Coronis System (Pérols, France) for monitoring and control applications in several environments such as homes and buildings [4]. The standard Wavenis, currently promoted and managed by Wavenis Open Standard Alliance, supports data rate up to $100 \mathrm{kbps}$ and adopts Gaussian frequency-shift keying (GFSK) modulation in conjunction with fast frequency hopping spread spectrum (FHSS). It defines the operations at the PHY, data link and NWK layers, delivered through proprietary APIs.

$M i W i$ wireless protocol [12], developed by Microchip Technology (Chandler, AZ, USA), uses low-power radio systems based on IEEE 802.15.4 for short-range transmissions. Given the small size of the protocol stack, MiWibased solutions are an alternative to ZigBee for low-cost applications requiring small memory space and able to operate on simple low-cost micro-controllers. The system is based on the IEEE 802.15.4 recommendations for wireless personal area networks (WPAN). It supports a smaller number of functions compared to ZigBee, and it is meant for simple networks with either peer-to-peer, star or mesh topologies in beacon-less configuration. MiWi provides advanced functionality at PHY, MAC, and NWK levels, all accessible through the use of proprietary APIs.

Insteon technology is developed by SmartLabs, Inc. (Irvine, CA, USA) and promoted by the Insteon Alliance for the field of home automation [13]. The system utilizes a dual technology to support communication between devices: it employs both powerline communications with X10 protocol and wireless communications using FSK modulation at $900 \mathrm{MHz}$. All Insteon compliant devices are peers, which means that each device is able to transmit, receive, and repeat any message compliant with the Insteon protocol, without the need of a master controller or routing software. The powerline communication infrastructure is used to provide synchronization to the wireless system.

$K N X-R F$ (KNX Association cvba, Diegem, Belgium) is a wireless solution specified in Supplement 22 of the KNX specification for cable based systems [14]. Thereby, $\mathrm{KNX}$ is not a protocol tailored for radio-frequency communication, but rather a home and building automation standard based on wired media that has been extended to support wireless communications. KNX RF operates at $868 \mathrm{MHz}$ using FSK modulation at $16.4 \mathrm{kbps}$. KNX RF allows unidirectional (transmit-only) devices in addition to conventional bidirectional ones. Transmit-only devices cannot be configured thorough the network. Data reliability is guaranteed only by APL layer acknowledgements, while link layer acknowledgments are forbidden. KNX RF does not provide any security mechanism. Since the transmitted data are neither encrypted nor subject to integrity check, KNX RF cannot fulfill the high demands of security in critical applications.

\section{WHAN architecture and MAC design}

In this section, we describe the proprietary MAC sublayer protocol developed on top of the SimpliciTI compliant PHY layer radio stack [15]. This is based on the low-power CC430 microcontroller system on chip (SoC) with integrated RF transceiver operating at $868 \mathrm{MHz}$ and low-power MSP430 controller [16]. The SimpliciTI system is here adopted for the intrusion detection experimental study as its wireless modules use a very basic core 
API compared to the other technologies listed in the previous section and thus allow for a more flexible network design. The proposed MAC sub-layer has a smaller code size ( 8 to $16 \mathrm{kB}$ ), and it allows to efficiently manage the different traffic patterns generated by the intrusion detection system, jointly exploiting both synchronized and nonsynchronized access schemes. The use of the ISM band $868-\mathrm{MHz}$ radio technology provides an improved robustness, compared to 2.4-GHz transceivers, against severe propagation conditions that are typical of indoor environments. This conclusion is confirmed by the experimental analysis illustrated in the next section.

The proposed network architecture, depicted in Figure 3, consists of one AP and a number of REs and EDs. The AP is the network coordinator which manages a low-power radio interface for two-way communications with the remote EDs and acts as a translator over any external network. For the APL layer, the AP node should guarantee the interoperability of the wireless infrastructure with other end-user operator services, i.e., portable human machine interfaces (HMI), radio-frequency identification (RFID), and video camera or thermocamera monitoring. As a consequence, the AP should perform the following tasks: (1) adaptively choose the network resources (through channel hopping and dynamic power control), (2) register new EDs (joining phase) and synchronize them to guarantee low duty cycle activity as prescribed by the standard [3], (3) periodically monitor the device status through standard-compliant keep-alive messages, and (4) guarantee real-time alarm message propagation with minimum latency.

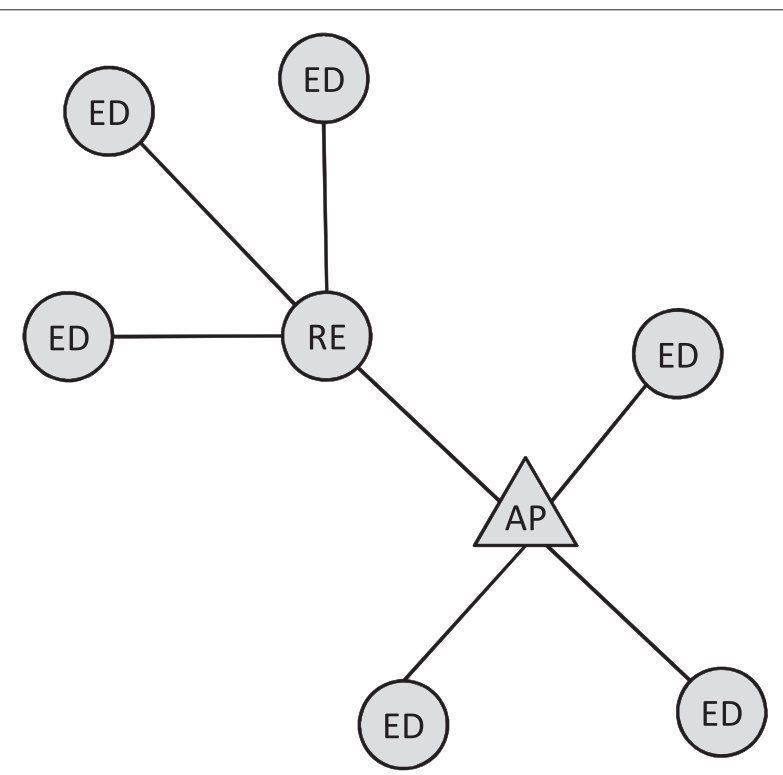

Figure 3 Node classes: end device (ED), access point (AP), range extender (RE).
The RE is the device responsible for multi-hop communications. Its basic function is to repeat the message from the ED under its control to the AP and vice versa. Finally, the ED is the low-power input/output wireless instrument that interacts with the sensor hardware to monitor the indoor environment and detect intrusions. Any ED should perform two functions: (1) periodic transmission of keep-alive messages containing basic information on radio device status (battery residual levels, receiver sensitivity, and channel quality), sensor status and tampering, and (2) transmission of alarm messages within a maximum latency of approximately $10 \mathrm{~s}$. Periodic retransmission of keep-alive messages conforms to the standard security grade 2 [3] that prescribes a minimum refresh rate of $20 \mathrm{~min}$.

\subsection{Network configuration}

The network configuration phase allows to register newcomer devices. Devices have to inform the AP about the role, type and number of their sensors in the network. The RE can join the network only by a direct connection. Instead, an ED device can join the network either with direct or indirect - through a RE - connection. In case of indirect connection, the ED communicates with a RE that forwards the message to/from the AP. Routing of packets is established at the time of network configuration.

\subsection{Frame structure and traffic management}

The MAC sub-layer developed for this experimental study proposes a frame structure that jointly handles the periodic traffic for monitoring the sensing device status and the potential bursty traffic for alarm message propagation in case of intrusion detection. The first task is implemented through a keep-alive message exchange with dedicated channel assignments, the second one by random slotted access through CSMA/CA.

The frame structure is shown in Figure 4. Time division duplex is employed to separate uplink and downlink, and to avoid transmission collisions. Logical frames have length $T_{\text {frame }}=208 \mathrm{~s}$ and consist of 64 time slots of $T_{\text {slot }}=3.25 \mathrm{~s}$ separated by guard times. The frame duration depends on the maximum number of EDs that can be assigned to the network, here set to 64 .

Periodic keep-alive message exchange session (of duration $T_{\text {frame }}$ ) starts with the transmission of the first keepalive message from ED \#1 (time slot 1) and stops with the last from ED \#64 (time slot \#64). Each time slot starts with the AP beacon message that contains the identifier (turn information or TI) of the device that has to update its status information. The beacon message is used for device synchronization following a similar approach as in [17] (see Section 3.4). The time slot is further divided into 65 mini-slots of duration $T=50 \mathrm{~ms}$, the first one being reserved for keep-alive message transmission (TI Answer) 


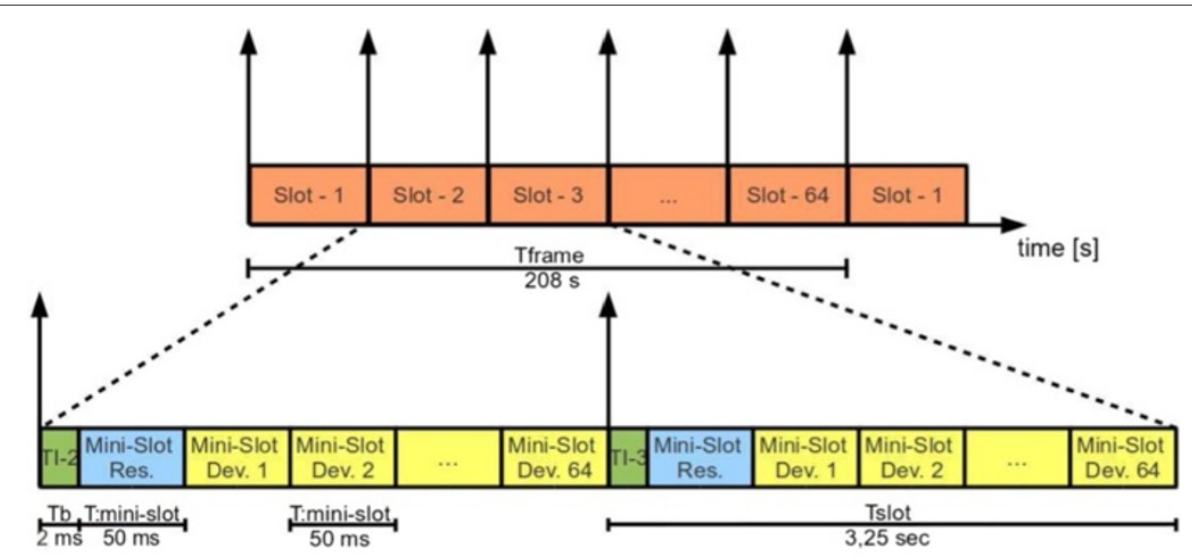

Keep Alive message structure

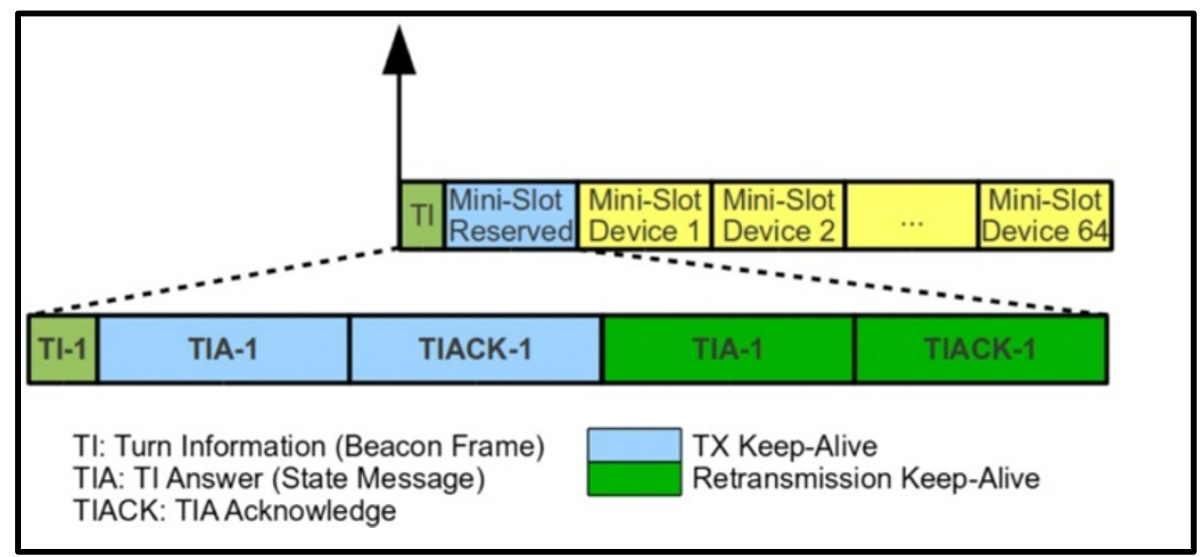

Figure 4 Frame structure and keep-alive message passing.

of the device indicated in the beacon message (holding the turn). As illustrated in Figure 4 (at bottom), a two-way explicit acknowledgement policy is adopted to guarantee reliable message delivery over links subject to fading. This ensures a sufficiently high level of channel immunity against short-term fluctuations of the channel gain. The remaining $64 \mathrm{mini}$-slots are used for synchronization operations and for alarm propagation as indicated below.

For the real-time alarm delivery, any ED detecting an intrusion cannot wait for its reserved mini-slot, it has to propagate the alarm message immediately after detection. The solution here proposed is to use a random access over the abovementioned 64 mini-slots. The ED willing to give the alarm overhears the beacon message (even if intended for another ED) to acquire synchronization, it randomly chooses one of the 64 mini-slots, and it uses carrier sensing before any transmission attempt to avoid cross-tier interference from external devices operating on the same frequencies (e.g., WiFi and Bluetooth). In the worst case, the overall latency of alarm message propagation is two times the slot duration, $2 T_{\text {slot }}=6.5 \mathrm{~s}$. If the first alarm transmission fails due to collision, the ED waits for a random period (equal to a number of mini-slots) before retransmission. If also the second one fails, the ED checks if the AP had changed channel by performing two retransmissions for each available channel until an ACK is observed.

\subsection{Interference-aware channel hopping}

To avoid bursty errors over consecutive polling sessions, a channel frequency hopping $(\mathrm{CH})$ phase is provided, adaptively initiated by the AP controller. $\mathrm{CH}$ is commonly adopted in industrial communications to increase robustness against interference and to provide an additional protection against eavesdroppers. The proposed system here uses two carrier frequencies defined in the sub- $\mathrm{GHz}$ band, namely 868 and $869 \mathrm{MHz}$. As shown in the message exchange example of Figure 5, the AP continuously monitors the background noise on the radio channel, and it might decide to perform a channel hopping in case of severe interference. At the first beacon signal lost, the ED programs a timer at $2 T_{\text {slot. }}$. If after the timer interval the ED does not receive the next beacon signal, the ED changes its working frequency accordingly. This waiting 

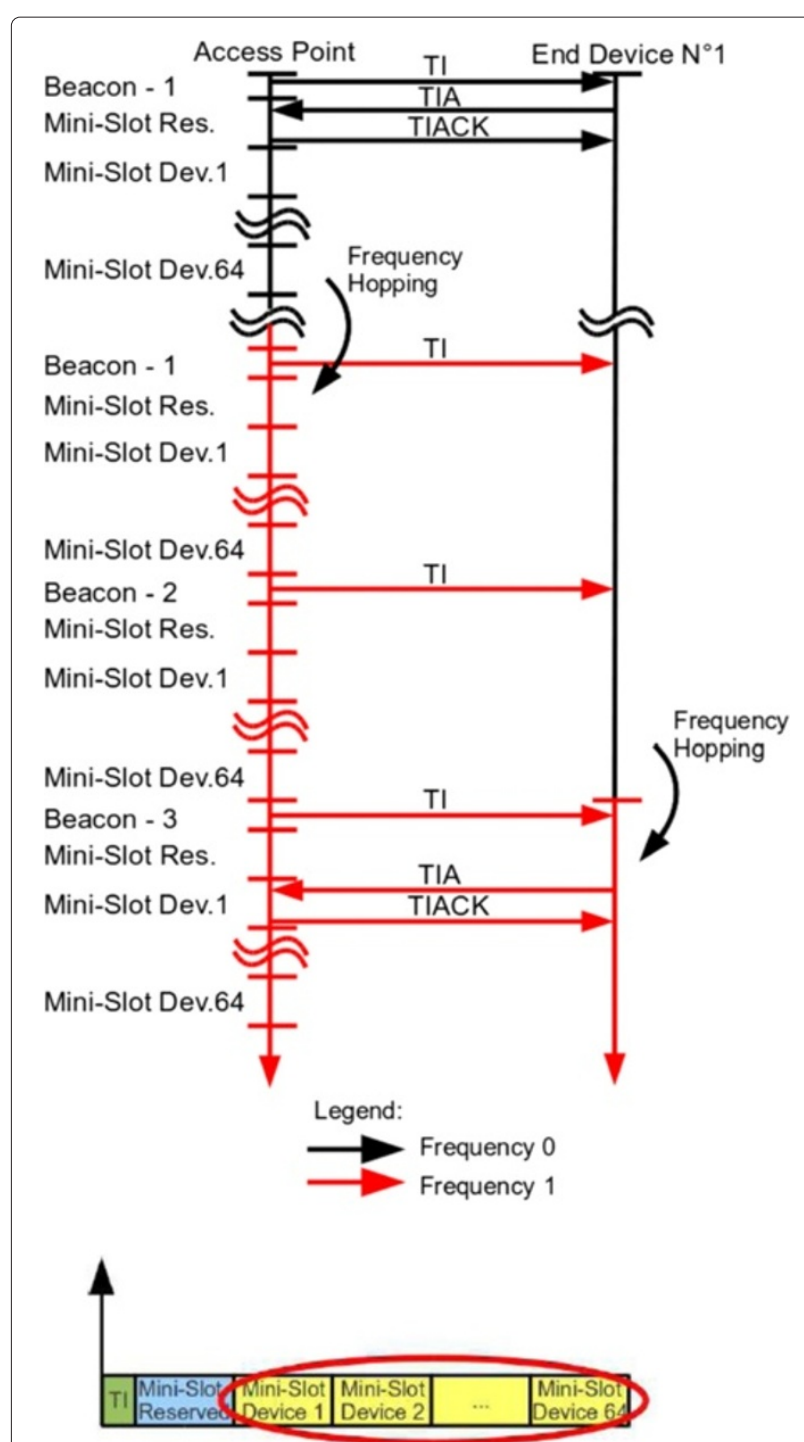

Figure 5 Message exchange during channel hopping phase.

period is necessary to be sure that beacon miss-detection is not caused by a temporary interferer.

\subsection{Duty cycling and device synchronization}

Device synchronization is based on the approach in [5]. A drift error compensation algorithm is developed to minimize idle listening and thus maximize the device lifetime. Each ED sleeps during the beacon transmissions intended for other devices and, excluding the alarm transmissions, it wakes up only to reply to its intended beacon message. The chosen interval $T_{\text {frame }}$ among successive beacon messages is large compared to typical re-synchronization intervals of 10 to $15 \mathrm{~s}$ [5]. Time-synchronized duty cycling is thus guaranteed by correcting and updating the sleep time $T_{\text {sleep }}$ (the time elapsed between two consecutive wake-up phases) on every new beacon message reception to account for the timing error experienced with the AP local oscillator. The proposed synchronization algorithm allows the ED to turn on the radio $T_{\mathrm{g}}=50 \mathrm{~ms}$ before the beacon message reception, with $T_{\mathrm{g}}$ being a pre-defined guard time. To account for the residual timing uncertainty after drift correction, the observed interval $\Delta t$ between the ED wake-up and the reception of the intended beacon message is modelled as Gaussian distributed with mean $T_{\mathrm{g}}=50 \mathrm{~ms}$ and maximum jitter of $4 \mathrm{~ms}$. Observed probability density function (pdf) is shown in Figure 6 at bottom. Given that the $k$ th beacon message is received, the ED device updates the sleep time $T_{\text {sleep }}(k)$ before the next wake-up by the following tracking approach

$$
T_{\text {sleep }}(k)=T_{\text {sleep }}(k-1)+\mu\left(\Delta t_{k}-T_{\mathrm{g}}\right),
$$

where $\Delta t_{k}=\Delta t(k)$ is the observed time between the ED wake-up and the reception of $k$ th beacon message. Initial value for $T_{\text {sleep }}$ assumes perfect synchronization as $T_{\text {sleep }}(0)=T_{\text {frame }}-T-T_{\mathrm{g}}$. The updating factor is set to $\mu=1 / 2$. As demonstrated in the experimental activity in Section 5, a larger guard time $\left(T_{\mathrm{g}}>50 \mathrm{~ms}\right)$ would cause unnecessary idle listening and higher energy consumption.

The tracking algorithm (1) can be applied when the intended $k$ th beacon message is correctly received: in Figure 6 top panel, this scenario is referred to as case 1 . On the other hand, case 2 refers to a scenario where the ED loses the beacon transmission due to a radio interference or a residual clock drift that delays its wakeup. In this case, tracking is not applied while an ad hoc re-synchronization policy is implemented. The ED stays awake to receive the next beacon transmission (intended for the ED holding the next turn). Next, to avoid interference with the transmission of the ED indicated in the beacon, it sends the keep-alive message using a reserved mini-slot chosen among the 64 available mini-slots (see also the framing structure in Figure 4) that follow the first mini-slot reserved for keep-alive message exchange (the reserved mini-slot for re-synchronization is assigned during the network configuration phase).

\subsection{Dynamic transmission power allocation}

A dynamic transmission power control algorithm is implemented to minimize the energy consumption during the periodic keep-alive message transmission. For each $\mathrm{ED}$, the AP controller reads the received signal strength (RSS) from the RSS indicator (RSSI) of the received TI answer message. The RSS value $\Gamma$ is embedded into the TI ACK (acknowledge) message and thus sent back to the corresponding ED. The ED uses this information to adapt the transmit power level $P_{\mathrm{T}}$ for the next keepalive message transmission. The goal of the power control 

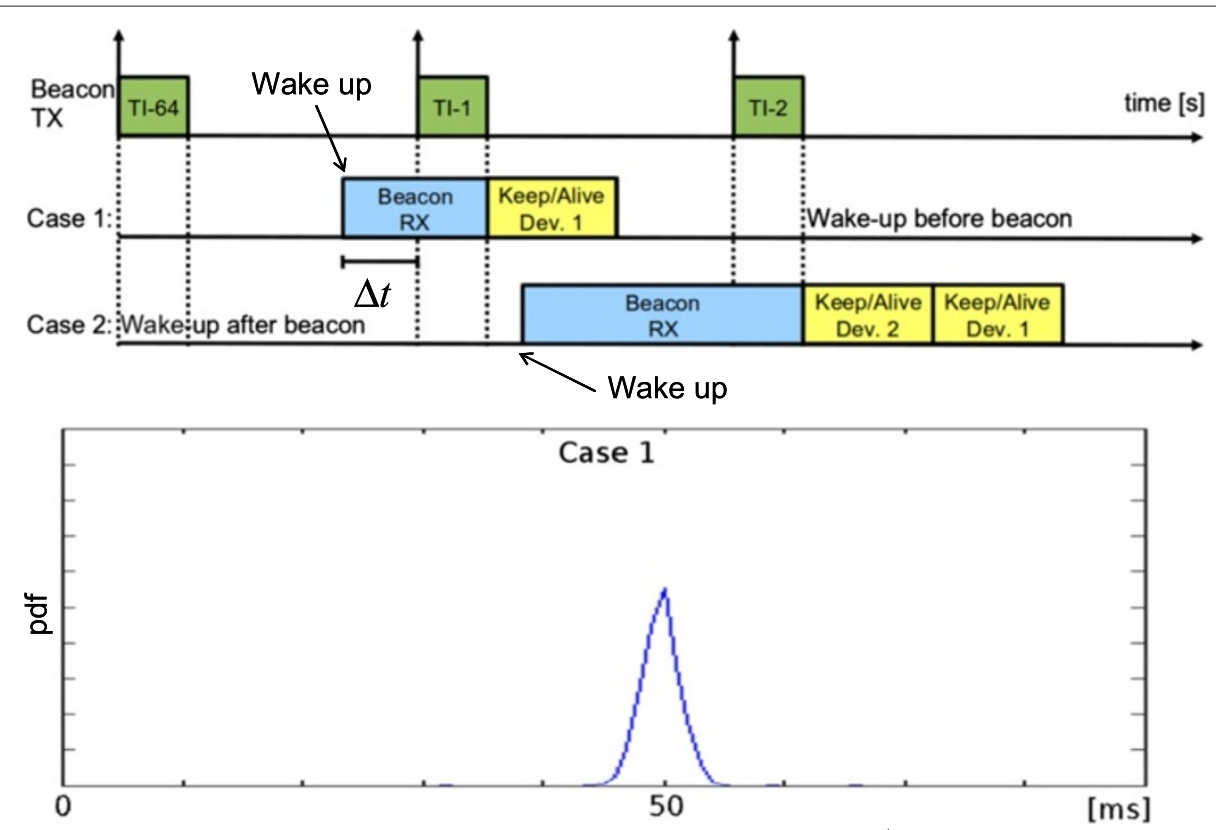

Time between wake up and beacon reception $\Delta t[\mathrm{~ms}]$

Figure 6 Device synchronization and timing error analysis. Top panel: device synchronization assuming two cases: wake-up before beacon reception (case 1) and wake-up after beacon reception (case 2). Case 2 requires re-synchronization using the subsequent beacon transmission. Bottom panel: probability density function of the time between the ED wake-up and the reception of beacon message (the average value of $50 \mathrm{~ms}$ equals to selected guard time $T_{\mathrm{g}}$, maximum jitter is $4 \mathrm{~ms}$ ).

algorithm is to adaptively adjust the power level $P_{\mathrm{T}}$ so that the RSS measurement $\Gamma$ can be kept within the range $\left(\Gamma_{\min }, \Gamma_{\max }\right)$. The two thresholds selected for the experimental activity in Section 5 are $\Gamma_{\max }=-20$ and $\Gamma_{\min }=-70 \mathrm{dBm}$.

\subsection{Encryption}

To improve the security level, all the transmissions are coded with the XTEA encoding scheme [18]. It is composed by three main elements which are a 128-bit encryption key, a 32-bit initialization vector, and a 32-bit counter. The initialization vector and the encryption key are set at built-time, while the counter value is determined at the time of the link creation between two or more devices. Devices that have formed the link preserve independent counters.

\section{A comparative study between $868-\mathrm{MHz}$ and 2.4-GHz RF technologies for WHAN}

Several standards for WHAN operate at $2.4 \mathrm{GHz}$. This frequency is also adopted in many indoor communication systems such as ZigBee, Wi-Fi, Bluetooth, and cordless phones. In this paper, we selected a radio technology in the $868-\mathrm{MHz}$ ISM band, as the use of sub$\mathrm{GHz}$ frequencies compared to $2.4 \mathrm{GHz}$ involves a twofold advantage, providing a low probability of interference and an increased radio coverage in harsh environments characterized by severe line-of-sight (LOS) blockage, due e.g., to walls and furniture in indoor environments. To verify this property, in this section, we present a comparative study between $868-\mathrm{MHz}$ and $2.4-\mathrm{GHz}$ radios based on a number of measurement campaigns carried out over long ranges in the indoor environment illustrated in Figure 7. The goal of the analysis is to demonstrate the importance of optimal frequency choice, taking into account the attenuation factors that highly affect the radiation inside buildings and thus have a crucial impact on network coverage.

\subsection{Channel modeling for WHAN}

The indoor wireless links without a clear LOS path undergo more severe power attenuations than those where the LOS path is fully unobstructed. This additional attenuation has been shown to be almost uncorrelated with the distance between transmitter and receiver [19]. The main scatterers responsible for the received power attenuation are mostly confined within the first and second Fresnel zones as these can be considered to contribute to the main wavefield energy [20]. For a wireless link where the direct path between transmitter and receiver has length $d$, the $n$th Fresnel zone is the region inside an ellipsoid with circular cross-section. The radius of the section at distance $q \leq d$ from the transmitter is [21]

$$
r_{n}(q)=\sqrt{n \lambda \frac{q(d-q)}{d}}
$$




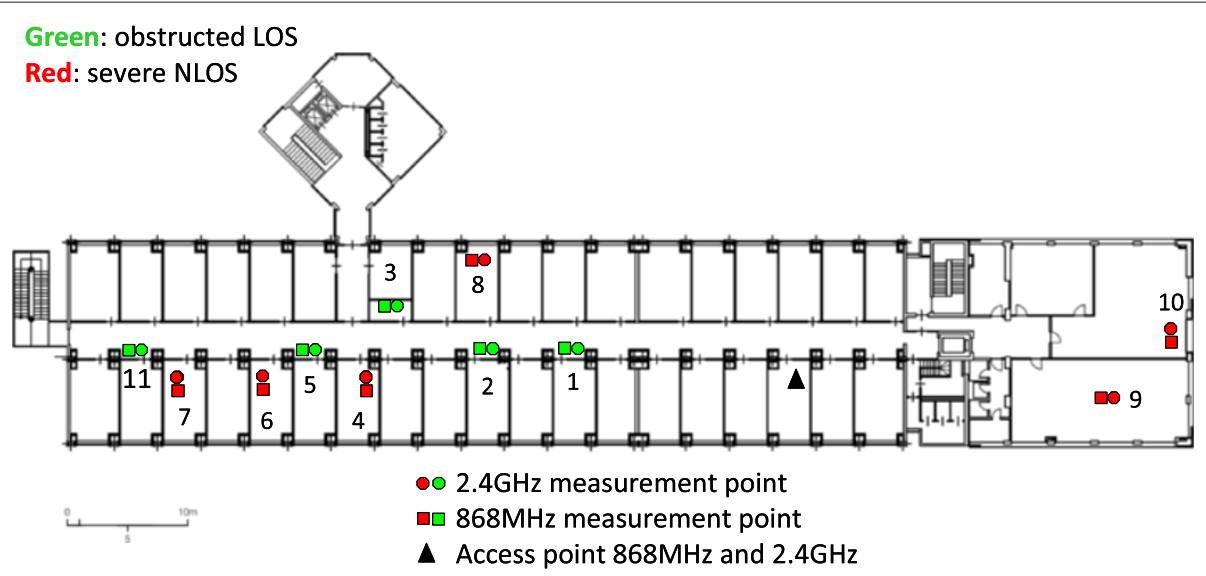

Figure 7 Comparative analysis between 2.4-GHz and 868- $\mathrm{MHz}$ technologies. Floor map of the indoor environment (third floor of DEIB building, Politecnico di Milano). Links corresponding to green positions are characterized by a partially obstructed LOS; links corresponding to red positions suffer from severe NLOS. The position of the AP is indicated by a triangular marker; rectangular and circular markers refer to the $868-\mathrm{MHz}$ and the 2.4-GHz co-located devices, respectively.

with $\lambda$ denoting the signal wavelength $(\lambda=0.125 \mathrm{~m}$ for $2.4 \mathrm{GHz}$ and $\lambda=0.345 \mathrm{~m}$ for $868 \mathrm{MHz}$ ).

We consider now the wireless link $\ell$ between any two transceivers of the WHAN, deployed at fixed locations and equipped with a single omnidirectional antenna. The RSS is the metric we employ to assess the quality of the radio link: it combines a LOS component and an excess attenuation that accounts for obstructions located within the Fresnel volumes, typically walls and furniture in the considered indoor scenario. The model for the RSS measured in logarithmic scale is [19]

$$
\gamma_{\ell}=\underbrace{g_{0}-10 \alpha \log _{10} \frac{d_{\ell}}{d_{0}}-\sigma_{\ell}}_{g_{\ell}}+s_{\ell}
$$

which consists of (1) a random Gaussian term $s_{\ell} \sim$ $N\left(0, v_{s}^{2}\right)$, with zero mean and standard deviation $v_{s}$, modeling the slow fading fluctuations of the received power due to people or objects moving in the area [22]; (2) a deterministic average term $g_{\ell}$ accounting for the freespace path loss with exponent $\alpha$ and the static multipath fading component [23] $\sigma_{\ell}$ acting as an additional attenuation caused by the fixed obstructions located within the Fresnel volume [24]. The term $g_{0}$ denotes the received power measured over free space (i.e., in LOS) at a reference distance $d_{0}$ (usually $d_{0}=3 \mathrm{~m}$ ).

The observed value for the path loss exponent can be reasonably set to $\alpha=2$ in short-range environments [19] where ground reflections can be neglected; this is the case for $d<d_{\mathrm{F}}$, with $d_{\mathrm{F}}=2 h_{\mathrm{tx}} h_{\mathrm{rx}} / \lambda$ denoting the Fresnel distance for antenna heights from the ground $h_{\mathrm{tx}}$ and $h_{\mathrm{rx}}$, at transmitter and receiver, respectively. Larger path loss exponent values, $\alpha>2$, are caused by reflections from the ground and can be experimented in long-range cases for $d>d_{\mathrm{F}}$.

\subsection{Radio equipment and test description}

In what follows, we describe the radio modules employed at the EDs for the comparative study on $868-\mathrm{MHz}$ and 2.4- $\mathrm{GHz}$ radio technologies. The module at $2.4 \mathrm{GHz}$ is detailed in [25]. The PHY layer is IEEE 802.15.4 standard compliant. The radio module provides different programmable high-power modes with maximum output power of $P_{\mathrm{T}}=18 \mathrm{dBm}$ and a digital RSSI that facilitates the implementation of the energy detection function. The demodulator is characterized by a minimum sensitivity of $\beta_{\min }^{(2.4)}=-98 \mathrm{dBm}$. The module is equipped with a lowpower 32-bit reduced instruction set computer (RISC) central processing unit (CPU) [25].

The 868-MHz module is part of Digi's XBee (Digi International, Minnetonka, MN, USA) family of RF products supporting FSK modulation and a fixed data rate of 24 kbps [26]. The receiver sensitivity is $\beta_{\min }^{(868)}=-112 \mathrm{dBm}$ with maximum programmable output power of $P_{\mathrm{T}}=25$ $\mathrm{dBm}$.

For the comparison, the two modules have been programmed to use the same output power, set to $18 \mathrm{dBm}$ so as to handle propagation loss over long ranges and possibly obstructed links. In both cases, we considered omnidirectional antennas with gain of $2 \mathrm{dBi}$. Antennas with such a gain can be commonly found on the market and do not require special alignments; the links used vertically polarized antennas.

The network layout consists of the above described radio modules and one AP collecting data simultaneously from both types of modules. These are co-located in the same enclosure and deployed in 11 different positions as 
shown in the floor map of Figure 7. For each position, the ED handling the two radio modules periodically sends message frames using alternatively the $2.4 \mathrm{GHz}$ and the $868 \mathrm{MHz}$ frequency every 2 s. On each received packet, the AP reads the RSSI on both frequencies to assess the link quality.

According to the beacon-less mode of the IEEE 802.15.4 MAC, during the 2.4-GHz network setup, the AP

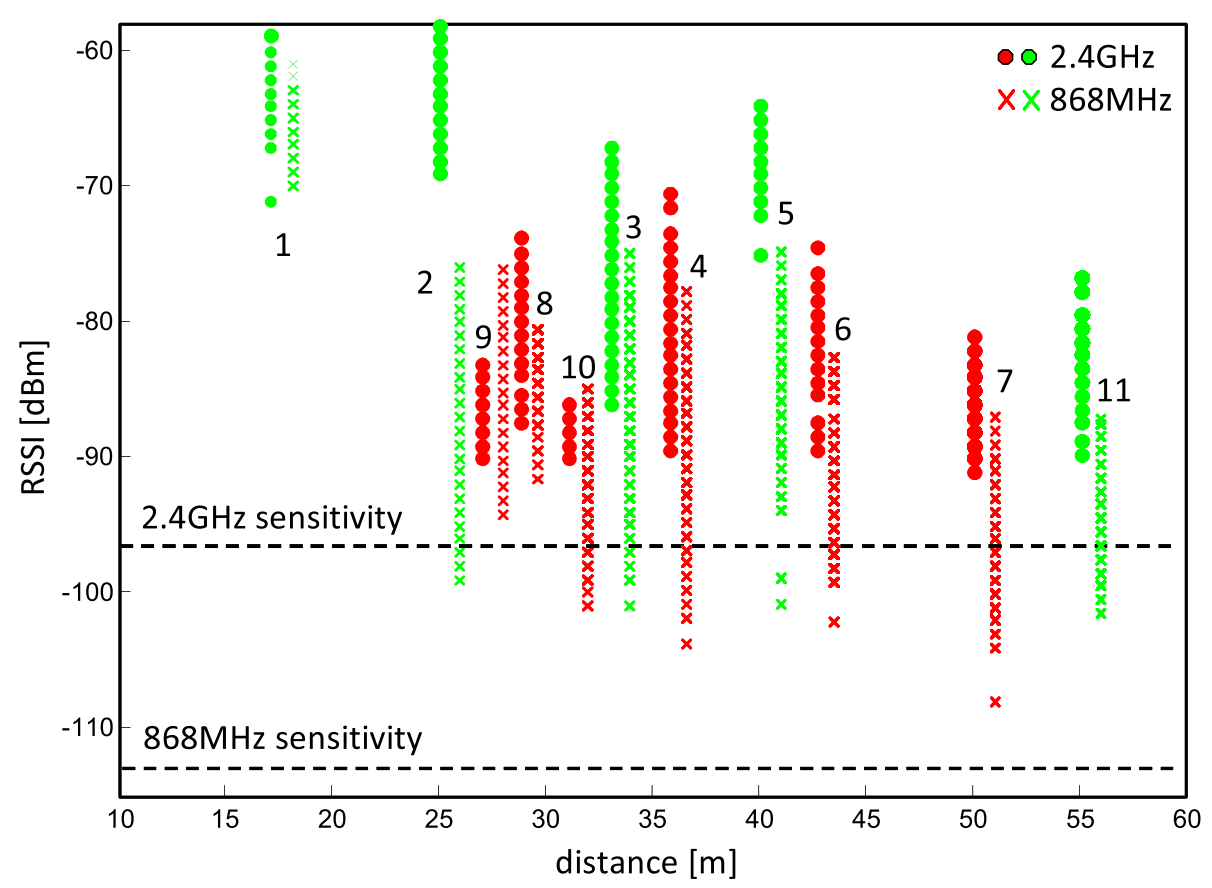

\begin{tabular}{|c|c|c|c|c|}
\hline \multirow{2}{*}{$\begin{array}{c}\text { Obstructed } \\
\text { LOS links }\end{array}$} & \multicolumn{2}{|c|}{$868 \mathrm{MHz}$} & \multicolumn{2}{c|}{$2.4 \mathrm{GHz}$} \\
\cline { 2 - 5 } & PER [\%] & $\begin{array}{r}\text { Avg. link } \\
\text { gain [dB] }\end{array}$ & PER [\%] & $\begin{array}{c}\text { Avg. link } \\
\text { gain [dB] }\end{array}$ \\
\hline 1 & $<5$ & 50 & $<5$ & 35 \\
\hline 2 & $<5$ & 29 & $<5$ & 28 \\
\hline 3 & $<5$ & 25.5 & $<5$ & 24 \\
\hline 5 & $<5$ & 26 & $<5$ & 32 \\
\hline 11 & $5-15$ & 20 & $5-15$ & 17 \\
\hline
\end{tabular}

\begin{tabular}{|c|c|c|c|c|}
\hline \multirow{2}{*}{$\begin{array}{c}\text { Severe } \\
\text { NLOS links }\end{array}$} & \multicolumn{2}{|c|}{$868 \mathrm{MHz}$} & \multicolumn{2}{c|}{$2.4 \mathrm{GHz}$} \\
\cline { 2 - 5 } & PER [\%] & $\begin{array}{c}\text { Avg. link } \\
\text { gain [dB] }\end{array}$ & PER [\%] & $\begin{array}{c}\text { Avg. link } \\
\text { gain [dB] }\end{array}$ \\
\hline 4 & $<5$ & 25 & $5-15$ & 21 \\
\hline 6 & $5-15$ & 18.5 & $>15$ & 12.5 \\
\hline 7 & $5-15$ & 14.5 & $>15$ & 11 \\
\hline 8 & $<5$ & 26 & $5-15$ & 20 \\
\hline 9 & $<5$ & 31 & $>15$ & 10 \\
\hline 10 & $5-15$ & 21 & $>15$ & 10 \\
\hline
\end{tabular}

Figure 8 RSS measurements and PER and link margin. Top panel: RSS measurements for 2.4-GHz and 868-MHz modules. Positions of EDs are defined in Figure 7 using the same color code. Bottom panel: PER and link margin for each position. 
optimally chooses the channel - out of the available 16 channels - by performing a passive scan to detect possible WiFi signals or co-channel interferers. Before sending data, the 2.4-GHz module of the ED implements an association MAC request to acquire the channel selected by the AP.

For the 2.4-GHz front end, the metric adopted to asses the channel quality is the link quality indicator (LQI). As verified experimentally, the LQI reading ranges from 0 to 255 , and it is used to compute the link gain $L_{\gamma}^{(2.4)}=$ LQI $\times \frac{85}{255}-7$ (see also [25]). The link gain metric $L_{\gamma}^{(2.4)}$ represents the RSS margin (in decibel scale) with respect to the receiver sensitivity $\beta_{\mathrm{min}}^{(2.4)}$ as

$$
L_{\gamma}^{(2.4)}=\gamma_{\ell}-\beta_{\min }^{(2.4)},
$$

where $\gamma_{\ell}$ is the observed RSS in (3).

Similarly, the link gain for the $868-\mathrm{MHz}$ radio module, $L_{\gamma}^{(868)}$, is computed from the RSSI reading $\gamma_{\ell}$ and the receiver sensitivity $\beta_{\min }^{(868)}$ as

$$
L_{\gamma}^{(868)}=\gamma_{\ell}-\beta_{\min }^{(868)} .
$$

The observed RSS $\gamma_{\ell}$ is directly obtained by reading the 8-bit RSSI register that monitors the automatic gain control state in the RX chain.

For each ED position and RF module, the values of the measured RSS samples are reported in Figure 8; the corresponding (average) link gains and the packet error rate (PER) $P_{\mathrm{E}}$ are illustrated in the table at bottom. Average link gains with respect to different channel realizations are computed as $\mathbb{E}\left[L_{\gamma}^{(868)}\right]$ for $868 \mathrm{MHz}$ and $\mathbb{E}\left[L_{\gamma}^{(2.4)}\right]$ for 2.4 GHz.

\subsection{Measurement analysis}

To highlight the impact of the propagation environment on the wireless system performance, in Figure 7 and 8, the positions and markers in green refer to the links characterized by obstructed LOS connection [20], while red markers indicates the links in severe NLOS condition where up to 15 walls are blocking the propagation. In what follows, the analysis focuses on the two propagation scenarios separately.

- Obstructed LOS links. The ED positions labeled as $\{1,2,3,5,11\}$ are located in an open area along a corridor: the links connecting the ED to the AP are obstructed by obstacles blocking the LOS path, while the first Fresnel volume (see Section 4.1) is only partially obstructed. For the relevant cases where the direct path distance is ranging between 25 and $60 \mathrm{~m}$ (positions $\{2,5,11\}$ ), both $2.4-\mathrm{GHz}$ and $868-\mathrm{MHz}$ radio links are characterized by comparable average link margins (notice that RSS measurements are affected by quantization noise and HW errors of up to $1 \mathrm{~dB}$ ) and PER values. These fall in the range $\mathbb{E}\left[L_{\gamma}^{(2.4)}\right]=17-32 \mathrm{~dB}$ for $2.4 \mathrm{GHz}$ and $\mathbb{E}\left[L_{\gamma}^{(868)}\right]=20-29 \mathrm{~dB}$ for $868 \mathrm{MHz}$.

- Severe NLOS links. The positions $\{4,6$ to 10$\}$ are located inside six different rooms at a distance from the AP of 25 to $40 \mathrm{~m}$. The corresponding links connecting to the AP can be classified as severe NLOS being the first Fresnel volume fully obstructed [19]. Obstructions for positions $\{4,6,7,8\}$ are characterized by walls 15 - to 25 -cm thick. The links connecting the EDs located in positions $\{9,10\}$ are obstructed by a metallic lift structure and walls of larger thickness. By analyzing the corresponding average radio link margins $\left(\mathbb{E}\left[L_{\gamma}^{(2.4)}\right], \mathbb{E}\left[L_{\gamma}^{(868)}\right]\right)$, it can be seen that the $868-\mathrm{MHz}$ radio links exhibit improved robustness against multipath effects. The observed link margins are larger compared to those observed for the 2.4- $\mathrm{GHz}$ radio modules as $\mathbb{E}\left[L_{\gamma}^{(868)}\right]>\mathbb{E}\left[L_{\gamma}^{(2.4)}\right]$. The average link margin increase $\mathbb{E}\left[\Delta L_{\gamma}\right]=\mathbb{E}\left[L_{\gamma}^{(868)}\right]-\mathbb{E}\left[L_{\gamma}^{(2.4)}\right]$ depends on the size of the obstructions, and it falls in the range $\mathbb{E}\left[\Delta L_{\gamma}\right]=3.5$ to $6 \mathrm{~dB}$ for links $\{4,6,7,8\}$ and $\mathbb{E}\left[\Delta L_{\gamma}\right]=11$ to $21 \mathrm{~dB}$ for positions $\{8,10\}$. The corresponding link margins observed for $2.4-\mathrm{GHz}$ links are $\mathbb{E}\left[L_{\gamma}^{(2.4)}\right]=11$ to $21 \mathrm{~dB}$ for positions $\{4,6,7,8\}$ and $\mathbb{E}\left[L_{\gamma}^{(2.4)}\right]=10 \mathrm{~dB}$ for positions $\{9,10\}$.

Link margins with $\mathbb{E}\left[L_{\gamma}^{(2.4)}\right] \leq 20 \mathrm{~dB}$ cause the PER to exceed the $15 \%$ limit. We can thus conclude that the $868-\mathrm{MHz}$ radio technology provides an improved robustness to NLOS propagation typical of indoor environments, and it is thus suited for WHAN applications. This motivated the employment of such a technology for the experimental analysis on the proposed WHAN in the next section.

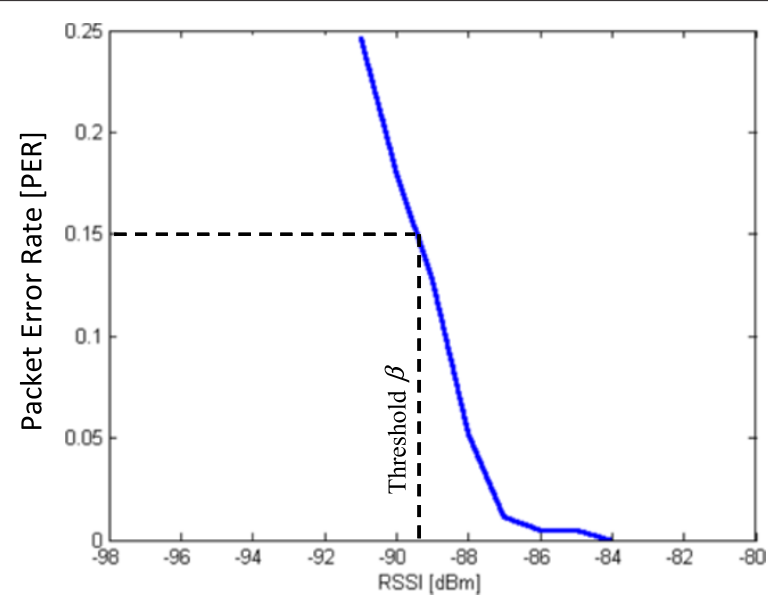

Figure 9 PER performance vs RSSI: experimental curve. 

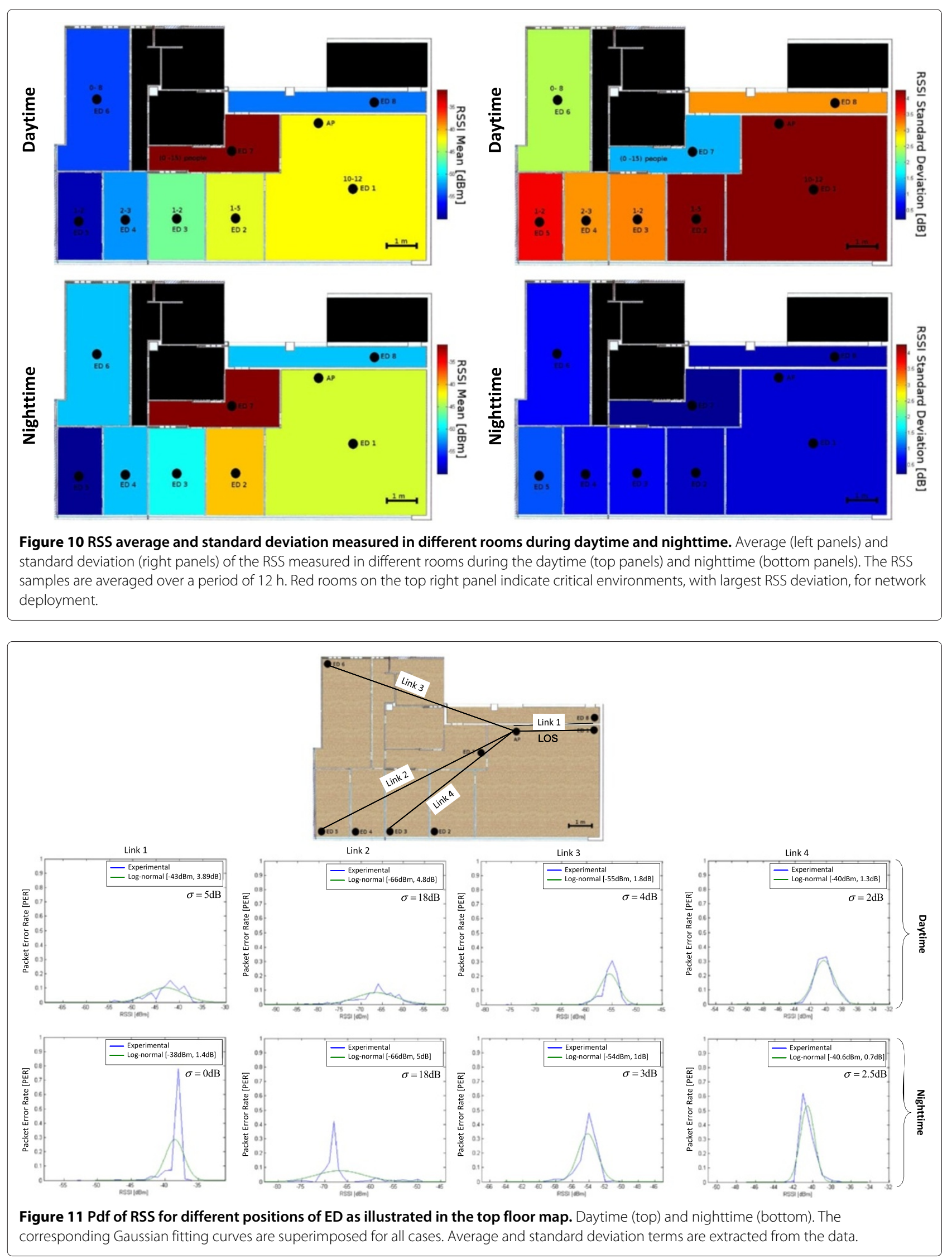


\section{Protocol testing and experimental activities}

The $868-\mathrm{MHz}$ radio technology adopted for testing the WHAN architecture and protocol proposed in Section 3 is part of the EM430F6137RF900 Texas Instrument kit with integrated SOC RF XCC430F6137IRGC. Key SOC features are as follows: (1) energy consumption from datasheet is characterized by a receive (RX) absorbed current of $15 \mathrm{~mA}$, while the transmit (TX) current is $33 \mathrm{~mA}$ for $P_{\mathrm{T}}=12 \mathrm{dBm}$ (maximum RF transmit power); (2) radio transceiver module is CC1101 [27] implementing 2-FSK as RF modulation with programmable data rate ranging from 0.6 to $600 \mathrm{kbps}$; (3) microcontroller unit (MCU) is MSP430 with advanced encryption standard (AES) compliant coprocessor, flash memory of $32 \mathrm{kB}, \mathrm{RAM} 4 \mathrm{kB}$, and ADC 12 bit.

Experimental activities for protocol testing have been conducted in two different indoor environments consisting of eight adjacent rooms. A single wireless ED was deployed in each room to monitor the surrounding area. People were moving inside each room causing random fluctuations of the radio signals. For all devices, the antenna height from the ground was below $1 \mathrm{~m}$. Propagation took place over a harsh radio environment with metallic objects (e.g., coaxial cabling, monitors/PCs, and tubes for air conditioning) and furniture causing significant attenuation.
Before presenting the protocol testing, the PER is evaluated as a function of the observed RSS. The PER depends on the degree of the RSS fluctuations: successful communication is considered when the RSS is above a threshold $\beta$ with probability

$$
P_{E}=\operatorname{Pr}\left[\gamma_{\ell} \geq \beta\right] .
$$

According to the experimental activity in Section 4, for 868-MHz EDs, the threshold is set to $\beta=\beta_{\min }^{(868)}+22$ $\mathrm{dB} \simeq-90 \mathrm{dBm}$, thus assuming a link margin of $L_{\gamma}^{(868)}=22$ $\mathrm{dB}$ above the minimum sensitivity $\beta_{\mathrm{min}}^{(868)}$ : this choice guarantees that $P_{E} \leq 5 \%$. Direct retransmissions in case of link failures further improve link reliability to a value that is consistent with grade 4 service (see Section 2.2). As depicted in Figure 9, any link experiencing $\gamma_{\ell}<\beta$ should be assumed as unreliable and is thus not considered during network planning.

\subsection{Channel measurements and network planning}

In this section, we analyze the impact of radio propagation at $868 \mathrm{MHz}$ on the wireless alarm message delivery. For the experimental activity, we used eight EDs Texas devices deployed in different rooms and one AP controller. The effects of propagation in each office room

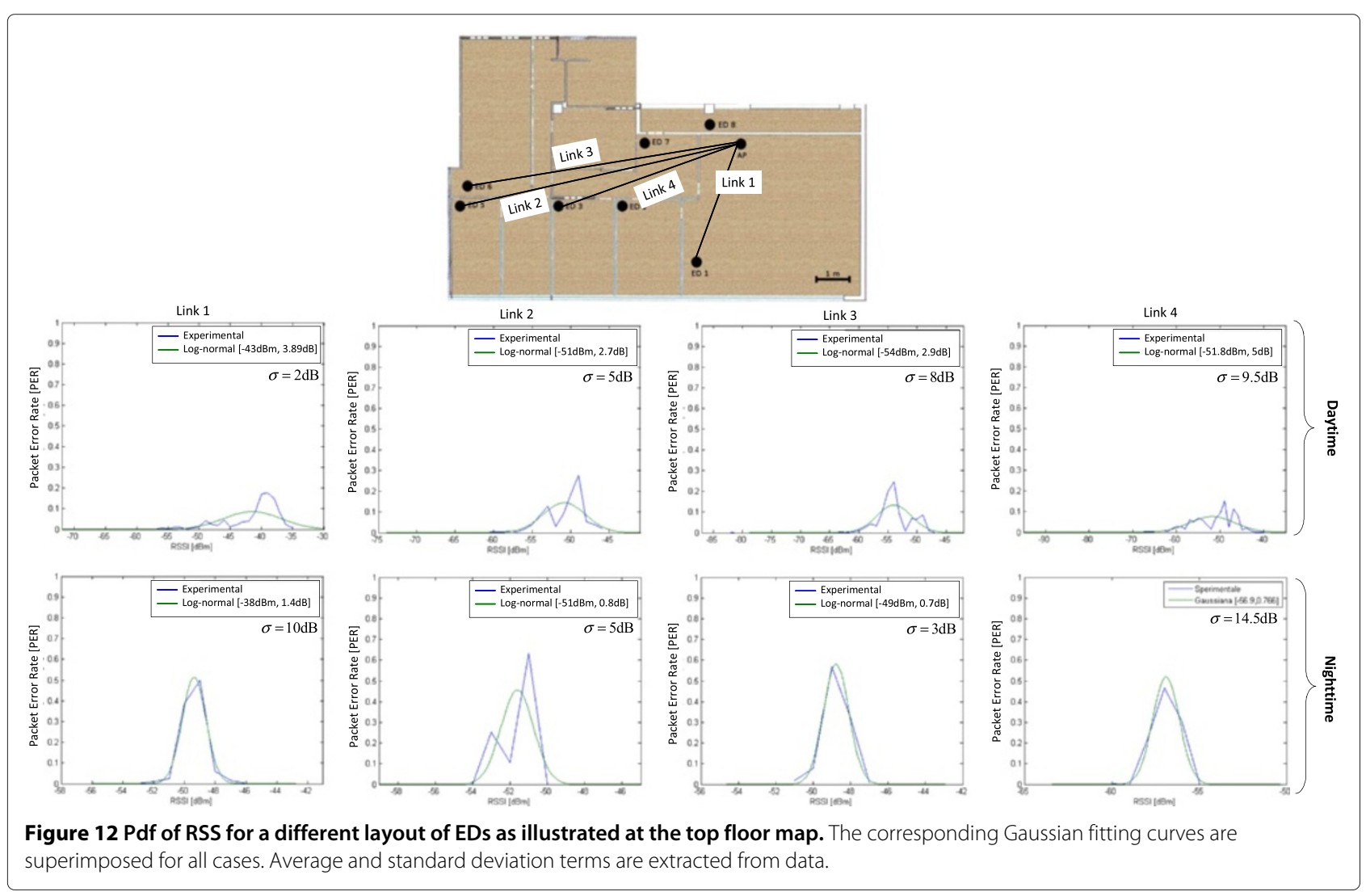


has been characterized by calculating the average and the standard deviation of the RSS samples measured over each AP-ED link. The RSS values can be read continuously from the RSSI status register [27].

The radio environment has been observed over a period of $48 \mathrm{~h}$, during which the position of the AP was fixed while the EDs were moved over two different locations per room every $24 \mathrm{~h}$ of operation. To highlight the impact of channel fluctuations, the transmit power is here set to $P_{\mathrm{T}}=12 \mathrm{dBm}$ while dynamic power allocation is disabled.

The resulting values of RSS average and standard deviation are shown in Figure 10. From the comparison between daytime (top panels) and nighttime (bottom panels), it is easy to notice that although the observed average RSS does not change significantly from daytime to nighttime, the standard deviation is strongly influenced by the presence of people in the environment during daytime [23]. The behavior of channel fluctuations is thus nonstationary as the degree of fluctuations in some rooms is shown to increase from about 0.5 to $1 \mathrm{~dB}$ in the nighttime to more than 4 to $5 \mathrm{~dB}$ in the daytime. This suggests to dynamically increase the transmit power during daytime by adding a 4- $\mathrm{dB}$ constant fade margin to compensate for the random signal strength fluctuations.

In Figures 11 and 12, the pdf of the RSS measurements is shown for different positions of the EDs, together with the Gaussian fitting curve according to model (3) and the observed attenuation $\sigma_{\ell}$ due to obstructions for each considered case. The average and standard deviation terms are extracted from the data: for most of the links, the fitting between the experimental curves and the Gaussian function is reasonably accurate. In all cases, the RSS standard deviation observed in the daytime is significantly greater than that in the nighttime, due to the effects of human body scattering $[28,29]$. This effect could also be observed by looking at Figure 13 showing the RSS measurements collected as a function of transmitter-receiver distance $d_{\ell}$ during daytime (top panels) and nighttime (bottom panels), respectively. The additional average signal attenuation compared to free-space propagation (for $\alpha=2$ in (3)) ranges between $\sigma_{\ell}=2 \mathrm{~dB}$ for obstructed LOS environments and up to $\sigma_{\ell}=14.5 \mathrm{~dB}$ under severe NLOS condition. Numerical results match also with previous analyses in the literature (see for example [6] to [30]) that predict RSS fluctuations in the interval 2 to $12 \mathrm{~dB}$.

\subsection{Analysis of network lifetime}

Figure 14 shows the energy consumption profile of a battery-equipped ED during a normal cycle of remote control. Measurements were obtained through an oscilloscope connected in parallel with the ED node itself. The absorbed current has been observed during five different phases: the wake-up period $\left(I_{\mathrm{wk}}=10 \mathrm{~mA}\right.$ with duration of $\left.T_{\mathrm{wk}}=4 \mathrm{~ms}\right)$, the receive period $\left(I_{\mathrm{RX}}=23 \mathrm{~mA}\right)$, the

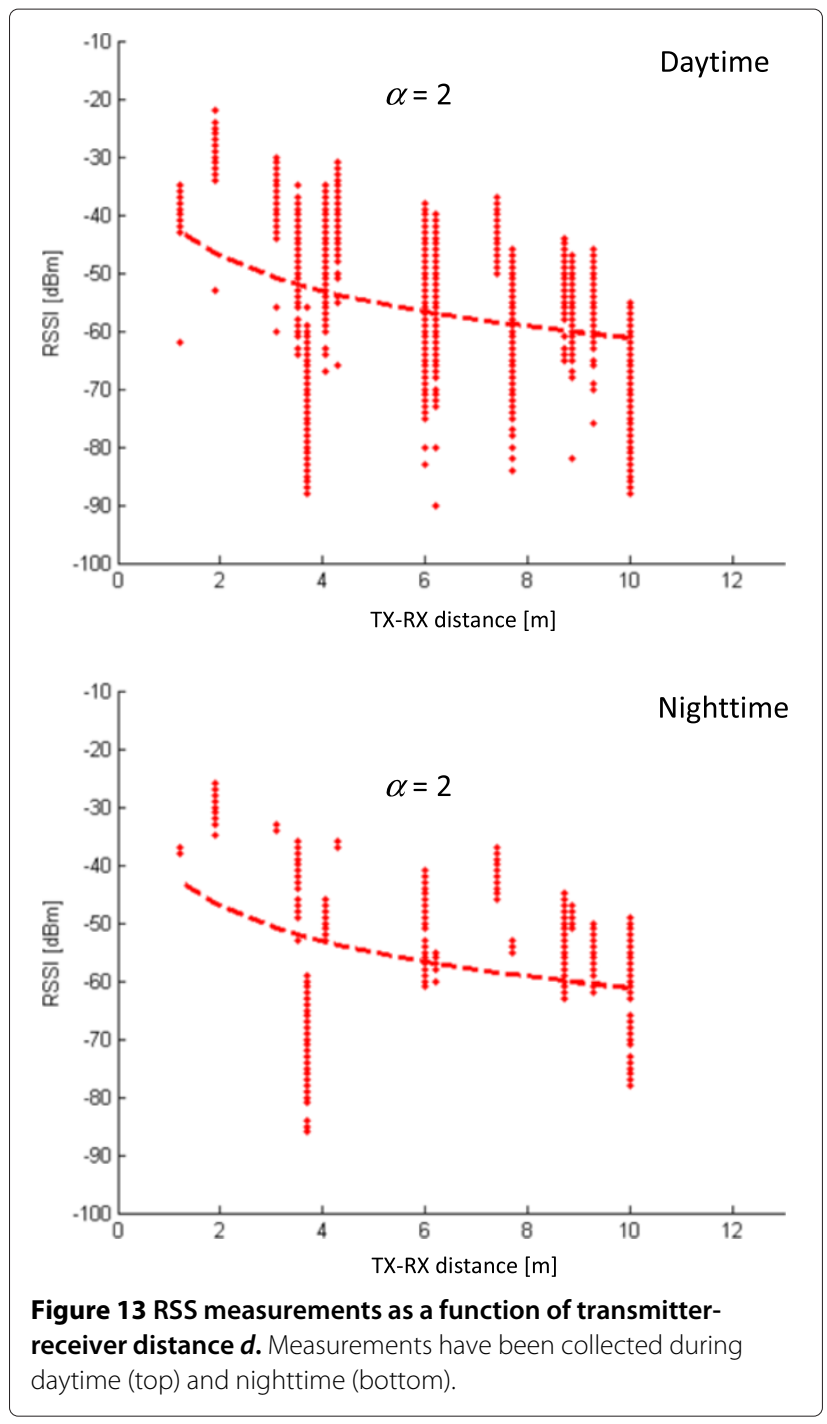

state message transmission $\left(I_{\mathrm{TX}}=40 \mathrm{~mA}\right.$ for a duration of $\left.T_{\mathrm{TX}}=2 \mathrm{~ms}\right)$, the acknowledge reception $\left(I_{\text {ack }}=26 \mathrm{~mA}\right.$ for a duration of $\left.T_{\text {ack }}=6 \mathrm{~ms}\right)$ and the sleep mode $\left(I_{\text {sleep }}=\right.$ $8 \mu \mathrm{A})$. To avoid the high background noise of the oscilloscope, the current absorbed during the sleep period was measured by a precision ammeter. The values measured during reception and sleep mode differ from the declared power consumptions, respectively $15 \mathrm{~mA}$ and $5 \mu \mathrm{A}$. In the sleep mode, the difference is due to the consumption of the wake-up timer not considered in the datasheet.

The average power absorbed by the ED has been calculated on every frame with the aim of drawing relevant considerations for network lifetime prediction. To allow for general insights, the power consumption is modeled as a function of the number of keep alive messages transmitted per frame, $\eta_{k_{-} \text {alive }}$, and the alarm transmission rate, $\eta_{\text {alarm }}$, defined as the average number of alarm messages per frame. 

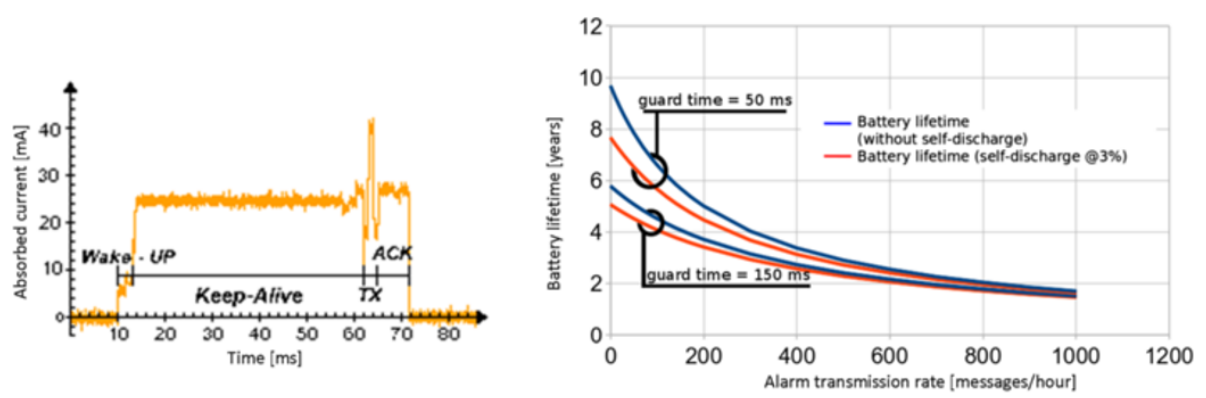

\begin{tabular}{|c|c|c|}
\hline Device State & Current Absorbed [mA] & Duration [ms] \\
\hline Wake-UP & 10 & 4 \\
\hline$R X$ (data/beacon) & 23 & $T_{\text {frem }}-T_{\text {slapp }}(k)$ \\
\hline$T X$ (data/beacon) & 40 & 2 \\
\hline RX ACK & 26 & 6 \\
\hline Sleep & 0.008 & n.a. \\
\hline
\end{tabular}

Figure 14 Energy consumption profile of a battery-equipped ED during a normal cycle of remote control. Left-side panel: measured absorbed current during wake-up, keep-alive receive (RX), and transmit (TX) phases (transmission of ACK message is included). Right-side panel: battery lifetime versus the number of transmitted alarm messages per hour. Guard time of $50 \mathrm{~ms}$ uses the proposed drift error compensation for prediction of timing uncertainty; the case of guard time $150 \mathrm{~ms}$ does not implement any error compensation. Bottom panel: energy consumption parameters.

For a given mini-slot duration $T$ and frame duration $T_{\text {frame }}$, the ED device has been designed to keep the radio transceiver active for receiving the beacon message, send the keep-alive message and the alarm message (if any). The average current absorbed $I_{\text {frame }}(k)$ at frame $k$ is thus

$$
\begin{aligned}
I_{\text {frame }}(k)= & \left(\eta_{k_{-} \text {alive }}+\eta_{\text {alarm }}\right) \frac{I_{\mathrm{TX}} T_{\mathrm{TX}}+I_{\mathrm{wk}} T_{\mathrm{wk}}}{T_{\text {frame }}} \\
& +\left(1-\frac{T_{\text {sleep }}(k)}{T_{\text {frame }}}\right) I_{\mathrm{RX}}+\frac{T_{\text {sleep }}(k)}{T_{\text {frame }}} I_{\text {sleep }},
\end{aligned}
$$

with $T_{\text {sleep }}(k)$ defined in (1). Taking into account the requirements of a grade 4 service that prescribes a duty cycle limitation of $1 \%$ measured over a cycle of $100 \mathrm{~s}$, the remote control by keep-alive message exchange is repeated about $\eta_{k_{-} \text {alive }}=17$ times per hour (corresponding approximately to 1 message per frame with a device duty cycle of $17 \times T_{\text {slot }} / 3,600 \simeq 0.01$ ). Therefore, the ED battery lifetime

$$
T_{\text {life }}=\frac{C_{\text {batt }}}{I_{\text {frame }}(k)+C_{\text {batt }} \xi_{\mathrm{d}}}
$$

can be estimated to a maximum of 9 years using a commercial $C_{\text {batt }}=1.4$ Ah lithium battery characterized by a self-discharge rate of $\xi_{\mathrm{d}}=0$ (per hour) and assuming as ideal case $\eta_{\text {alarm }}=0$ alarm messages/hour, $T_{\text {sleep }}(k) \simeq$ $T_{\text {sleep }}(0)=T_{\text {frame }}-T-T_{\mathrm{g}}, \forall k$, and a guard time of $T_{\mathrm{g}}=50 \mathrm{~ms}$.
In Figure 14, the impact on the expected ED lifetime of the alarm transmission rate $\eta_{\text {alarm }}$, the battery selfdischarge rate $\xi_{\mathrm{d}}$ (for $\xi_{\mathrm{d}}=0$ and $\xi_{\mathrm{d}}=3 \%$ per year) and the choice for the guard time $T_{\mathrm{g}}$ is analyzed. We considered two cases: in the first one, drift compensation is employed according to the specifications of the proposed MAC sub-layer with guaranteed guard time of $T_{\mathrm{g}}=50$ $\mathrm{ms}$; the second case refers to the use of a guard time of $T_{\mathrm{g}}=150 \mathrm{~ms}$ without clock drift compensation. The use of the proposed drift compensation strategy allows significant gains in network lifetime that reaches up to two times for alarm transmission rate below the practical case of $\eta_{\text {alarm }} \leq 200$ messages/hour (corresponding to $200 \times T_{\text {frame }} / 3,600$ messages per frame).

\section{Conclusion}

In this paper, a proprietary MAC link-layer protocol tailored for smart surveillance and intrusion detection applications has been developed on top of the SimpliciTI compliant radio stack [15] using the ultra-low-power CC430 microcontroller [16]. The use of the ISM band 868 $\mathrm{MHz}$ for cable replacing in home automation provides an improved robustness to NLOS propagation typical of indoor environments, and it is therefore the most suitable choice for smart ambient surveillance. The proposed protocol uses a very basic core API, allowing for a more flexible network design. The medium access scheme has been designed to jointly exploit both scheduled and random access to guarantee low-power periodic keep-alive message exchange and real-time alarm propagation with minimum latency, respectively. The analysis of battery 
consumption proved that low-power duty cycling can guarantee a lifetime of several years. Channel characterization, radio coverage, network planning, and power control have also been discussed by experimental results in indoor environments, showing that the proposed system is a promising platform for WHAN applications.

\section{Competing interests}

The authors declare that they have no competing interests.

\section{Acknowledgements}

The work has been supported partially by the European Research Project Dense Cooperative Wireless Cloud Network (DIWINE) under FP7 ICT Objective 1.1 - The Network of the Future.

\section{Author details}

${ }^{1}$ Dipartimento di Elettronica, Informazione e Bioingegneria (DEIB), Politecnico di Milano, Piazza Leonardo da Vinci 32, 20133 Milano, Italy. ${ }^{2}$ Comelit R\&D s.r.l., via Quinto Alpini 6/A, 24124 Bergamo, Italy. ${ }^{3}$ Institute of Electronics, Computer and Telecommunication Engineering (IEIIT), Italian National Research Council (CNR), Milan site, c/o DEIB Politecnico di Milano, via Ponzio 34/5, 20133 Milano, Italy.

Received: 25 January 2013 Accepted: 21 October 2013

Published: 14 January 2014

\section{References}

1. AJD Rathnayaka, VM Potdar, SJ Kuruppu, Evaluation of wireless home automation technologies, in Proceedings of the 5th IEEE International Conference on Digital Ecosystems and Technologies (Daejeon, Korea, 31 May-03 June 2011)

2. M Spadacini, S Savazzi, M Nicoli, S Nicoli, Wireless networks for smart surveillance: Technologies, protocol design and experiments, in Proceedings IEEE Wireless Communications and Networking Conference, Workshop on Internet of Things Enabling Technologies (Paris, 1 Apr 2012)

3. European Standard, EN 50131-5-3, Alarm systems - Intrusion systems, Part 5-3: Requirements for interconnections equipment using radio frequency techniques. (CEN, Brussels, 2005)

4. C Gomez, J Paradells, Wireless home automation networks: A survey of architectures and technologies. IEEE Commun. Mag. 48(6), 92-101 (2010)

5. W Ye, J Heidemann, D Estrin, An energy efficient MAC protocol for wireless sensor networks. IEEE INFOCOM. 3, 1567-1576 (2002)

6. C Reinisch, W Kastner, G Neugschwandtner, W Granzer, Wireless technologies in home and building automation, in Proceedings IEEE International Conference on Industrial Informatics (Vienna, 23-27 June 2007)

7. N Sriskanthan, F Tan, A Karande, Bluetooth based home automation system. Microprocess. Microsy. 26(6), 281-289 (2002)

8. K Gill, S-H Yang, F Yao, X Lu, A ZigBee-based home automation system. IEEE Trans. Consum. Electron. 55(5), 422-430 (2009)

9. IEEE, Standard IEEE 802.15.4-2006, Part 15.4: Wireless medium access control (MAC) and physical layer (PHY) specifications for low-rate wireless personal area networks (LR-WPAN). (IEEE, 2006)

10. T Jorgensen, NT Johansen, Z-wave as home control RF platform, http:// www.zen-sys.com/. Accessed Sept 2012

11. J Ploennigs, U Ryssel, K Kabitzsch, Performance analysis of the EnOcean wireless sensor network protocol, in Proceedings of the IEEE Conference on Emerging Technologies and Factory Automation (ETFA) (Bilbao, 13-16 Sept 2010)

12. D Flowers, Y Yang, Microchip MiWi Wireless Networking Protocol Stack. (Microchip Technology, Chandler, AZ, USA, 2010)

13. RJ Robles, TH Kim, A review on security in smart-home development. Int. J. Adv. Sci. Technol. 15(2), 13-22 (2010)

14. W Granzer, F Praus, W Kastner, Security in building automation systems. IEEE Trans. Ind. Electron. 57(1), 3622-3630 (2010)

15. L Friedman, SimpliciTI: Simple Modular RF Network Specification. (Texas Instruments, Inc., San Diego, CA, USA, 2009)

16. Texas Instruments, Data-sheet, MSP430 TMSOC with RF Core. (Texas Instruments, Inc., San Diego, CA, USA, 2010)
17. S Ganeriwal, I Tsigkogiannis, H Shim, V Tsiatsis, MB Srivastava, D Ganesan, Estimating clock uncertainty for efficient duty-cycling in sensor networks. IEEE/ACM Trans. Netw. 17(3), 843-856 (2009)

18. MN Roger, DJ Wheeler, Tea extensions. Technical Report, Computer Laboratory, University of Cambridge, 1997

19. DJ Lee, WC Lee, Propagation prediction in and through buildings. IEEE Trans Vehicular Technol. 49(5), 1529-1533 (2000)

20. S Savazzi, S Guardiano, U Spagnolini, Wireless sensor network modeling and deployment challenges in oil and gas refinery plants. Int. J. Distrib. Sens. N (2013). doi:10.1155/2013/383168

21. SR Saunders, AA Zavala, Antennas and Propagation for Wireless Communications Systems, 2nd edn. (Wiley, New York, 2007)

22. OW Ata, AM Shahateet, MI Jawadeh, Al Amro, An indoor propagation model based on a novel multi wall attenuation loss formula at frequencies $900 \mathrm{MHz}$ and $2.4 \mathrm{GHz}$. Wireless Pers. Commun. 69(1), 23-26 (2012). doi:10.1007/s11277-012-0558-x

23. P Castiglione, S Savazzi, M Nicoli, T Zemen, Partner selection in indoor-to-outdoor cooperative networks: An experimental study. IEEE J. Selected Areas Commun. 31(8), 1559-1571 (2013)

24. WC Lee, DJ Lee, Microcell prediction in dense urban area. IEEE Trans. Vehicular Technol. 47(1), 246-253 (1998)

25. NXP Laboratories, Data-sheet JN-DS-JN5148-001, IEEE 802.15.4 Wireless Microcontroller JN5148-001. (NXP Laboratories, UK, 2012)

26. Digi International Inc., XBee-PRO 868 RF Modules, Product Manual, v1x6x XBee-PRO 868, 90001020_B, Digi International Inc.11001 Bren Road East Minnetonka. http://www.digi.com, Accessed Feb 2011

27. Texas Instruments, Data-sheet CC1101, Low-Power Sub-1 GHz RF Transceiver. (Texas Instruments, CA, USA, 2012)

28. S Savazzi, M Nicoli, F Carminati, M Riva, A Bayesian approach to device-free localization: modeling and experimental assessment. IEEE J. Sel. Topics Signal Proc (2014). doi:10.1109/JSTSP.2013.2286772

29. M Cheffena, Physical-statistical channel model for signal effect by moving human bodies. EURASIP J. Wireless Commun. Netw. 77(1) (2012). doi:10.1186/1687-1499-2012-77

30. F Gustafsson, F Gunnarsson, Mobile positioning using wireless networks: possibilities and fundamental limitations based on available wireless network measurements. IEEE Signal Process. Mag. 22(4), 41-53 (2005)

doi:10.1186/1687-1499-2014-6

Cite this article as: Spadacini et al:: Wireless home automation networks for indoor surveillance: technologies and experiments. EURASIP Journal on Wireless Communications and Networking 2014 2014:6.

\section{Submit your manuscript to a SpringerOpen ${ }^{\circ}$ journal and benefit from:}

- Convenient online submission

Rigorous peer review

- Immediate publication on acceptance

- Open access: articles freely available online

- High visibility within the field

- Retaining the copyright to your article

Submit your next manuscript at $>$ springeropen.com 